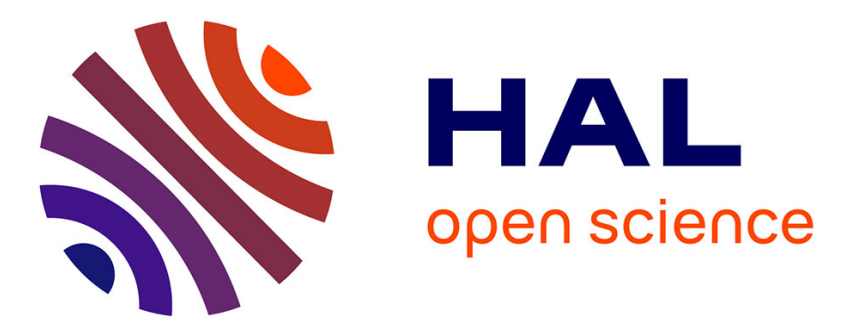

\title{
A 3D mechanical model of the early mammalian embryo
}

Laurent Le Guillou, Nicolas Dard, Jérôme Glisse, Bernard Maro, Sophie

Louvet-Vallée, Bertrand Laforge

\section{To cite this version:}

Laurent Le Guillou, Nicolas Dard, Jérôme Glisse, Bernard Maro, Sophie Louvet-Vallée, et al.. A 3D mechanical model of the early mammalian embryo. Journal of Biological Physics and Chemistry, 2009, 9, pp.11. in2p3-00453960

\section{HAL Id: in2p3-00453960 https://hal.in2p3.fr/in2p3-00453960}

Submitted on 8 Feb 2010

HAL is a multi-disciplinary open access archive for the deposit and dissemination of scientific research documents, whether they are published or not. The documents may come from teaching and research institutions in France or abroad, or from public or private research centers.
L'archive ouverte pluridisciplinaire HAL, est destinée au dépôt et à la diffusion de documents scientifiques de niveau recherche, publiés ou non, émanant des établissements d'enseignement et de recherche français ou étrangers, des laboratoires publics ou privés. 


\title{
A 3D mechanical model of the early mammal embryo
}

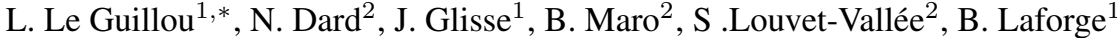 \\ ${ }^{1}$ UPMC Univ. Paris 06, UMR 7585, Laboratoire de Physique Nucléaire et des Hautes Energies (LPNHE), F-75005 Paris, France \\ ${ }^{2}$ UPMC Univ. Paris 06, UMR 7622, Laboratoire de Biologie Cellulaire du Développement, F-75005 Paris, France \\ *corresponding author, e-mail: 1lg@lpnhe.in2p3.fr
}

\begin{abstract}
The early development of the mammalian embryo leads to the formation of a structure composed by an outer layer of polarized cells surrounding an inner mass of non-polarized cells. Experimental biology has shown that this organisation results from changes in cell polarity, cell shape and intercellular contacts at the 8 and 16cell stages. In order to examine how the physical properties of embryo cells (adhesion, cortical tension) influences the organisation of the cells within the embryo, our team has developed a 3D mechanical model of the dividing early embryo, based on cellular Potts models. In this paper we will present the principles of our simulations, the methodology used and we will show that a very simple mechanical model can reproduce the main structural features (geometry, cell arrangement) of the mammalian embryo during its early developmental stages, up to the 16-cell stage.
\end{abstract}

Keywords: embryogenesis, cellular Potts model, stochastic simulation

\section{Cell differenciation during the early develop- ment of the mammalian embryo}

During the mammalian pre-implantation development, the fertilized oocyte undergoes several divisions (Fig. 1), but cells remain undifferentiated up to the late 8-cell stage. At the 16-cell stage, two distinct cell populations are observed for the first time, an outer layer of polarized blastomeres surrounding non-polarized inner cells. It is known since the late 60 s that the position of a cell within the embryo strongly influences its phenotype and its fate $[16,19]$. The phenotypic divergence between inside and outside cells results from the polarization of blastomeres at the 8-cell stage during the process of compaction and from asymmetric cell divisions during the following mitosis; these asymmetric divisions lead to the inheritance of the apical domain by the outside daughter cell $[2,9]$. During the next division, cell shape seems to play an important role in this process, controlling the ratio of inside/outside cells at 32-cell stages $[4,10,15]$. Cell shape is partly determined by the mechanical constraints applied on each cell: the embryo organization thus seems to result from an interplay between cell internal structure, cortical tension, cell-cell contacts, cell adhesive properties and asymmetric cell divisions.

The role of cell shape is particularly emphasized when we consider the evolution of embryos arising from a single 2-cell blastomere. Such embryos develop as the normal ones, except that they are smaller in size: when they are composed of 16 blastomeres, these half embryos are at the same molecular stage than a normal 32cell embryo, but they exhibit a very different ratio of inside/outside cells compared to a normal one [3]. As only cell geometry is different between half embryos and normal ones, these data clearly point out the importance of cell shape and mechanical interactions within the embryo.

To assess the role of mechanics during the early developmental stages, and particularly to be able to test different hypotheses regarding the relationship between the mechanical interactions within the embryo and the cell lineage divergence, we decided to develop a simple 3D mechanical model of the mammalian embryo,
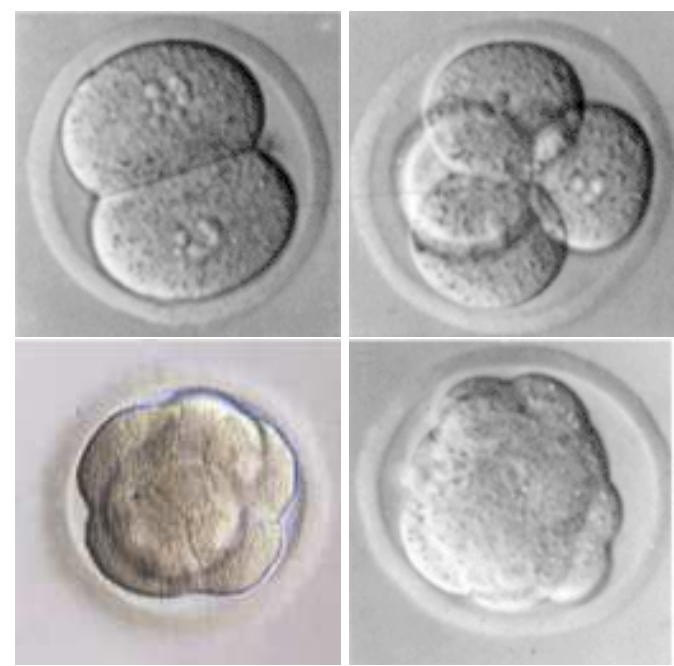

Figure 1. Mammalian embryo (Mus musculus) developmental stages. Top left: embryo at 2-cell stage. Top right: 4-cell stage. Bottom left: late 8-cell stage, compaction has occured, the rounding effect is clearly visible. Bottom right: 16-cell stage. 


\section{Computational model}

\subsection{Cellular Potts model}

Our mechanical model is based on a cellular Potts model (CPM), a popular lattice-based stochastic model for the simulation of morphogenesis processes. Within this framework, biological mechanisms are described in terms of effective interactions and constraint energies [5], and it is relatively easy to model the mechanical behavior of multicellular tissus and organisms, taking into account cell elasticity, cell adhesion, mitosis and haptotaxis. Originally developed to explain cell sorting in terms of differential adhesion [6-8], cellular Potts models have since been successfully used to model several morphogenesis problems, like the motion of retina cells in the chicken embryo [17], the formation of the fruiting body of Dictyostelium discoideum [13, 14], the cell patterning in the Drosophila retina [11], and the morphogenesis of the chicken limb [18].

The cellular Potts model is lattice-based: space is divided in individual sites (analogous to spins in the original Potts model) lying on a lattice, and each lattice site $(x, y, z)$ has an associate index value $\sigma(x, y, z)$; a domain of connected sites with the same index represents an embryo cell (or the surrounding medium). A hamiltonian $H_{\text {Potts }}$ (representing the free energy of the system) is associated to any lattice configuration, and $H_{\text {Potts }}$ is usually written as $[5,6]$ :

$$
\begin{gathered}
H_{\text {Potts }}=\alpha \sum_{(x, y, z)}^{\text {sites }} \sum_{\left(x^{\prime}, y^{\prime}, z^{\prime}\right)}^{\text {neighbors }}\left[1-\delta_{\sigma(x, y, z), \sigma\left(x^{\prime}, y^{\prime}, z^{\prime}\right)}\right] \\
+\quad \text { additional energy terms }
\end{gathered}
$$

where the additional terms express the various surface constraints or volume constraints one wants to include in a model. In fact, the first term of this hamiltonian is an estimator of the surface energy associated to all the cell-cell interfaces; hence, this term is a way to quickly estimate the area $S_{i, j}$ of each cell-cell or cell-medium interface between cells/medium $i$ and $j$ (Fig. 2):

$$
\begin{aligned}
S_{i, j} \simeq K \sum_{(x, y, z)} \sum_{\left(x^{\prime}, y^{\prime}, z^{\prime}\right)}^{\text {sites }} & {\left[1-\delta_{\sigma(x, y, z), \sigma\left(x^{\prime}, y^{\prime}, z^{\prime}\right)}\right] } \\
& \times \delta_{i, \sigma(x, y, z)} \delta_{j, \sigma\left(x^{\prime}, y^{\prime}, z^{\prime}\right)}
\end{aligned}
$$

where the pre-factor $K$ has to be calibrated to obtain the expected surface to volume ratio. This area estimator has the advantage to be easily computed with a very low cost in terms of CPU.

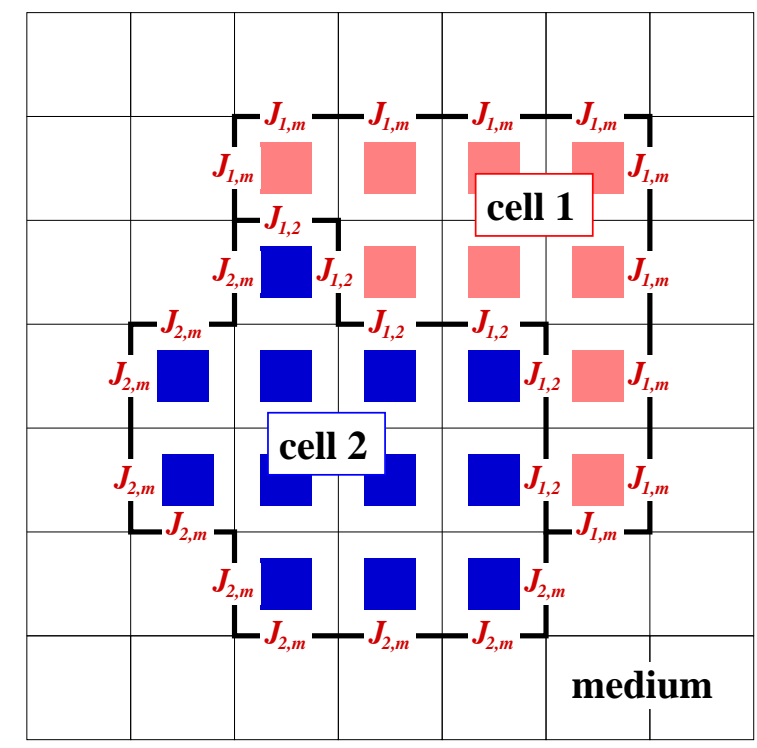

Figure 2. Surface energy term in the cellular Potts model.

For our mammal embryo model, we wish to preserve cell volumes and approximatively preserve surface areas; therefore, the hamiltonian of our model is written as:

$$
\begin{aligned}
H_{\text {Potts }}= & \sum_{i}^{\text {cells }} \lambda_{i}^{B}\left[V_{i}-V_{i}^{B}\right]^{2}+\sum_{i}^{\text {cells }} \lambda_{i}^{C}\left[S_{i}-S_{i}^{C}\right]^{2} \\
& -\sum_{i, j \neq i}^{\text {interfaces }} J_{i, j} S_{i, j}
\end{aligned}
$$

In this equation, the first two terms roughly describe the elastic properties of all cells. The first term somehow describes the compressibility of each cell: $\lambda_{i}^{B}$ is the bulk stiffness of cell $i, V_{i}$ its current volume, and $V_{i}^{B}$ its target volume; this term prevents cell disappearance. The second term in (3) represents the cell membrane elasticity: $\lambda_{i}^{C}$ being the cortical stiffness of cell $i, S_{i}$ its current surface area, and $S_{i}^{C}$ its target area; this term control the shape of each cell. The last term represents the adhesive properties between cells. For each interface between two cells $i$ and $j$ (or one cell and the medium), $S_{i, j}$ is the contact area, and $J_{i, j}$ the associated energy cost. Since this term is accounted negatively, $J_{i, j}>0$ means that cell $i$ and cell $j$ are adhesive and tend to increase their contact surface $S_{i, j}$. The $J_{i, j}$ adhesion coefficients are symmetric.

The system evolution is driven by a Metropolis algorithm: cell shapes and positions evolve through a stochastic process, where the system tries to minimize its total free energy $[5,6]$. At each simulation step, a 
lattice site $(x, y, z)$ is randomly chosen; its index value is changed into the index value of one of its first-order neighbors; the energy variation $\Delta H$ induced by this local change is then computed, and this change is accepted with the probability $P_{\text {flip }}$ defined as:

$$
P_{\text {flip }}= \begin{cases}1 & \text { if } \Delta H<0 \\ e^{-\Delta H / k T} & \text { otherwise }\end{cases}
$$

where $k T$ gives the magnitude of the allowed energy fluctuations, thus representing the fluctuations of the position of the physical cell membranes. Using the usual terminology for CPM models, one Monte Carlo Step (MCS) corresponds to $N$ flip attempts, where $N$ is the total number of sites in the simulation lattice.

\subsection{Control of grid anisotropy effects}

As we want to predict with some accuracy the shape of all cells, we use a quite high spatial sampling: for instance, the first embryo cell has a diameter of 80 to 160 in lattice units (corresponding to a length scale of 1 to $0.5 \mu \mathrm{m}$ per lattice site). With such a sampling, some grid anisotropy effects may affect the behavior of the model: without any precaution, intercellular frontiers perpendicular to the lattice main axes will be accounted with a slightly lower surface energy than other frontiers, thus favorising the apparition of unrealistic cellular shapes.

We tried to limit this effect in two ways: first, when computing the surface area estimator given by (2), we considered lattice neighbors up to the fifth-nearest neighbors (56 neighbors in 3D). Second, we changed the definition of the surface area estimator to the following one:

$$
S_{i, j} \simeq K \sum_{(x, y, z)} \sum_{\substack{\left(x^{\prime}, y^{\prime}, z^{\prime}\right) \\ \times w_{\mathrm{r}} \times \delta_{i, \sigma(x, y, z)} \delta_{j, \sigma\left(x^{\prime}, y^{\prime}, z^{\prime}\right)}}}^{\text {neighbors }}\left[1-\delta_{\sigma(x, y, z), \sigma\left(x^{\prime}, y^{\prime}, z^{\prime}\right)}\right]
$$

where $w_{\mathrm{r}}$ weights the contribution of the neighbors of rank $r$, with $r=1,2,3,4,5$. As we use neighbors up to the $5^{\text {th }}$ rank, we optimized the weights $\left\{w_{r}\right\}_{r=1 \ldots 5}$ to limit the grid anisotropy effects.

Due to the high spatial sampling and to our modifications of the Potts surface area estimator, we wrote our own optimized cellular Potts model engine, named Bobbles [12]. We adapted it to be able to easily run our simulations on the computer farm of the IN2P3 computing center (CC-IN2P3) and on the GRID network, to take advantage of the computing power available there for high energy physics experiments.

\section{Mechanical model of the early embryo}

\subsection{Embryo, cells and medium}

In our model, we describe the evolution of the mammalian embryo, starting from the zygote enclosed inside the protective envelope named zona pellucida $(\mathrm{ZP})$, up to the 16-cell stage.

Each individual cell $i$ is described by 4 independent parameters : $\lambda_{i}^{B}, V_{i}^{B}, \lambda_{i}^{C}, S_{i}^{C}$. But in practice, setting the proper stiffness $\lambda_{i}^{B}, \lambda_{i}^{C}$, the target volume $V_{i}^{B}$ and the target area $S_{i}^{C}$ in order to get a cell of a given diameter at equilibrium is not trivial. It is much more practical to directly specify the radius of a cell.

Under most common conditions in a cellular Potts model, an isolated cell is spherical at equilibrium; it hamiltonian is:

$$
H_{i}=\lambda_{i}^{B}\left[V_{i}-V_{i}^{B}\right]^{2}+\lambda_{i}^{C}\left[S_{i}-S_{i}^{C}\right]^{2}
$$

and its equilibrium radius can be computed by solving the following equation,

$$
\frac{\partial H_{i}}{\partial r}\left(r=r_{0}^{i}\right)=0
$$

The solution is given below by (8).

It is then possible to define a cell $i$ by a new set of 4 parameters : its equilibrium radius (when isolated) $r_{i}^{0}$, the energy scale of its elastic properties $E_{i}^{0}$, and two adimensional elastic coefficients $\eta_{i}$ and $\nu_{i}$. These new parameters are linked to $\lambda_{i}^{B}, V_{i}^{B}, \lambda_{i}^{C}$ and $S_{i}^{C}$ by the following equations:

$$
\left\{\begin{aligned}
\lambda_{i}^{B} & =\frac{9 E_{i}^{0}}{16 \pi^{2}\left(r_{i}^{0}\right)^{6}}=\frac{E_{i}^{0}}{\left(V_{i}^{0}\right)^{2}} \\
\lambda_{i}^{C} & =\frac{9 \nu_{i} E_{i}^{0}}{16 \pi^{2}\left(r_{i}^{0}\right)^{4}} \\
V_{i}^{B} & =\frac{4}{3} \pi\left(r_{i}^{B}\right)^{3} \\
r_{i}^{B} & =r_{i}^{0}\left(\frac{A_{i}^{1 / 3}}{2}+\frac{8 \nu_{i}^{2} \eta_{i}^{4}}{A_{i}^{1 / 3}}-2 \nu_{i} \eta_{i}^{2}\right) \\
S_{i}^{C} & =4 \pi\left(r_{i}^{C}\right)^{2} \\
r_{i}^{C} & =\eta r_{i}^{B} \\
A_{i}= & 4+24 \nu_{i}-64 \nu_{i}^{3} \eta_{i}^{6}+ \\
& 4 \sqrt{1+12 \nu_{i}-32 \nu_{i}^{3} \eta_{i}^{6}+36 \nu_{i}^{2}-192 \nu_{i}^{4} \eta_{i}^{6}}
\end{aligned}\right.
$$


With these new parameters, we may rewrote the cell hamiltonian as:

$$
\begin{aligned}
H_{i}(r)=E_{i}^{0} \times( & {\left[\left[\frac{r}{r_{i}^{0}}\right]^{3}-\left[\frac{r_{i}^{B}}{r_{i}^{0}}\right]^{3}\right]^{2}+} \\
& \left.9 \nu_{i}\left[\left[\frac{r}{r_{i}^{0}}\right]^{2}-\left[\frac{r_{i}^{C}}{r_{i}^{0}}\right]^{2}\right]^{2}\right)
\end{aligned}
$$

In our simulations, all the cells of the same type and at the same developmental stage have the same simulation parameters. Except during mitosis, the target volume and target area do not change during the simulation, as no cell growth occurs during the embryo segmentation process.

Cell adhesive properties and cortical tension are represented in our model through the $J_{i, j}$ coefficient. Changing its value allows to simulate differential adhesion effects like cell-sorting [6], and is also helpful to induce a compaction-like effect (see below).

The whole embryo is surrounded by the zona pellucida, defined in our model as a spherical shell made of an inert medium fixed in the simulation lattice. It is assumed that the $\mathrm{ZP}$ is not adhesive, and rigid; this last approximation is acceptable before the blastocoele cavity formation.

The extracellular medium is traited the same way than all cells, but with infinite target volume and area, and no associated energy cost:

$$
\left\{\begin{array}{l}
V_{m}^{0}=\infty \quad S_{m}^{0}=\infty \\
\lambda_{m}^{B}=\lambda_{m}^{C}=0 \\
J_{i, m}=0 \quad \forall i
\end{array}\right.
$$

\subsection{Mitosis}

During the early stages of the development of the mammalian embryo, cell divisions are asynchronous, but all cells divide roughly every 12 hours, approximatively within one hour interval. The detailled cell division process cannot be properly described in the framework of such a simple mechanical model. In our simulations, mitosis is implemented in a straightforward manner. At each development stage, the following process is repeated, until all cells have undergone mitosis: (1) a cell is selected randomly, and the orientation of the mitotic spindle is randomly chosen. Between 8- and 16-cell stages, the direction of the mitotic spindle is chosen randomly using an experimental angular distribution [3]; (2) a plan passing through the cell barycenter and perpendicular to the mitotic spindle direction is defined; (3) the lattice sites belonging to the cell undergoing mitosis on one side of the plan are attributed to a newly created cell; (4) the target volume and target area of the two daughter cells are updated, to insure that the equilibrium volume is divided by 2 to preserve the total volume of all embryo cells;(5) the simulation runs 1000 MCS to reach the next mechanical equilibrium.

Just before mitosis, each cell tends to become less adhesive to its neighbors and tends to round up; however we did not take this into account for now in our model.

\subsection{Compaction}

In the late 8-cell stage, the embryo undergoes a process named compaction: all cells polarize along an apicobasal axis, and their basolateral membrane becomes strongly adhesive compared to the apical one. This leads to the flattening of cells upon one another; as a whole, the embryo looks much more spherical. The flattening is thought to be related to changes in the properties of E-cadherin and its distribution along the apical and basolateral domains [21].

To describe this process in our mechanical model, we increase the cell-cell adhesion through the adhesion terms $J_{i, j}$. Doubling this coefficient is enough to observe a morphological transformation similar to the one observed in vivo: the whole embryo becomes much more spherical. It should be noted that there is an interplay between the cell elasticity coefficients $E_{i}^{0}, \eta_{i}, \nu_{i}$ and the cell-cell adhesion term $J_{i, j}$ : the same rounding effect may be obtained either by increasing the adhesion term $J_{i, j}$ or the elastic coefficient $\eta_{i}$, or by decreasing $E_{i}^{0}$ or $\nu_{i}$.

\section{Results}

Using our model, we ran several 3D simulations with different parameter sets. We were able to reproduce the observed evolution of the embryo with a good qualitative agreement, at all developmental stages from the 1-cell to 16-cell stage, including the compaction (See Fig. 3). Simulation results were checked visually and compared to video microscopy and to confocal images to assess the realism of the model.

The main qualitative features (embryo shape, cell arrangement) are quite insensitive to the exact values of the chosen simulation parameters; we interpret this result as an evidence of the robustness of our model and its ability to describe the mechanical aspects of the evolution of the early mammalian embryo. Of course, the exact 
position, shape and cell arrangement depend on the simulation parameters, as well as the inside/outside ratio at the 16-cell stage. Hence the need of a proper calibration of our model which is described in the next section.

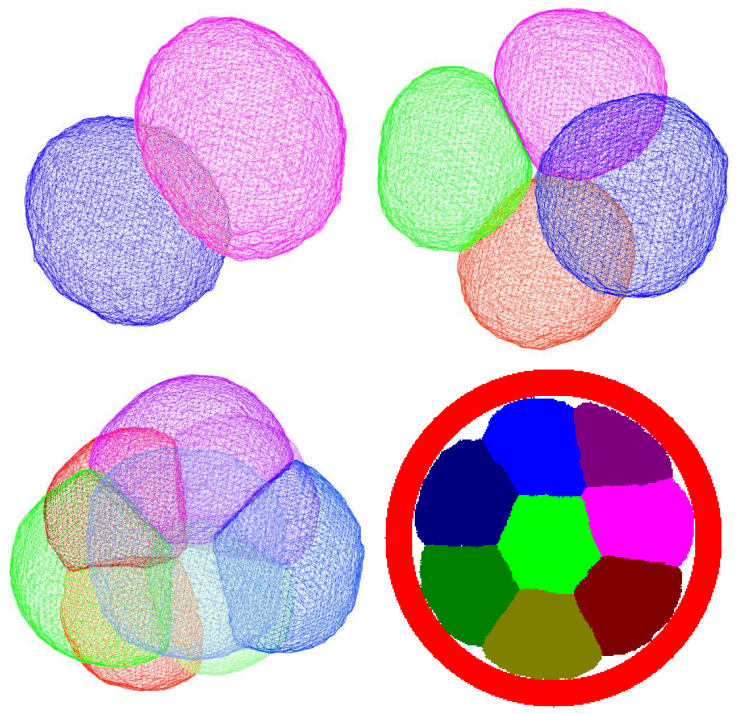

Figure 3. Simulation of the mammalian embryo at various stages. Top left: embryo at 2-cell stage (3D view, only frontiers are represented using the "Marching Cubes" algorithm, and the zona pellucida (ZP) surrounding the simulated embryo is not shown). Top right: same embryo at 4-cell stage. Bottom left: late 8-cell stage, compaction is occuring, the cell flattening is clearly visible. Bottom right: A slice of the 16-cell stage, in order to better see the cell arrangement inside the embryo. The zona pellucida is drawn in red. This simulation has been done on a $128^{3}$ lattice, and took 16000 MCS from stage 1-cell to stage 16-cell. Simulation parameters: $128^{3}$ lattice, $k T=1.0$, $E_{0}=600.0, r_{\text {zygote }}^{0}=56.0, \eta=0.4, \nu=2.0, r_{z p}=60.0$, $J_{c, c}^{[1-8]}=10.0, J_{c, c}^{[8-16]}=20.0$.

\section{Model calibration with confocal imaging}

In order to give some predictive power to our mechanical model, not only in terms of general embryo shape and cell arrangement, but to be able to predict, at least statistically, the precise cell shapes, the expected contact angles, and the ratio of internal/external cells at 16-cell stage, both for control embryo and artificially modified ones, we need to properly calibrate the model on existing data. As this work is still in progress, in this section we will mainly outline the procedures we have developed for this calibration task.

In addition to cell volume and surface estimations, our calibration procedures take advantage of the Young's re- lation between surface tensions computed at each contact point between 3 or 4 cells. At a contact point between 3 cells $A, B, C$ (or 2 cells and the extracellular medium), the mechanical equilibrium can be expressed as (See Fig. 4 and Fig. 5):

$$
\vec{\gamma}_{A B}+\vec{\gamma}_{A C}+\vec{\gamma}_{B C}=\overrightarrow{0}
$$

where $\gamma_{i, j}$ is the surface tension between cell $i$ and cell $j$. An equivalent relation can be written at 4-cell contact points.

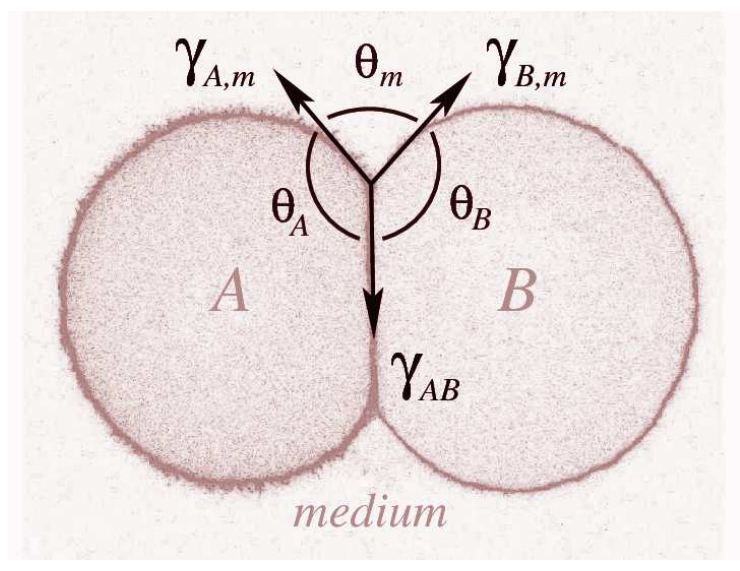

Figure 4. Calibration using confocal images (only one z-slice is shown). At this 2-cell stage, angle measurements at the contact point between cell $A$, cell $B$ and the extracellular medium provide numerical constraints on the model parameters. $\gamma_{A, m}$, $\gamma_{B, m}$ and $\gamma_{A, B}$ are the surface tensions at the interfaces between, respectively, cell $A$ and the medium, cell $B$ and the medium, and between cells $A$ and $B$.

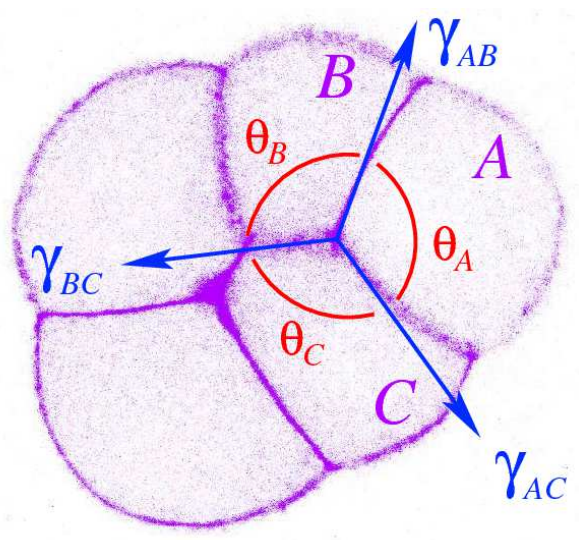

Figure 5. Calibration using confocal images. At this 8-cell stage only 5 cells are visible on this z-slice. $\gamma_{A B}, \gamma_{A C}$ and $\gamma_{B C}$ are the surface tensions at the interfaces between, respectively, cells $A$ and $B, A$ and $C$, and $B$ and $C$. 
In our model, surface tensions may be derived from the general hamiltonian of the system given in (3):

$\gamma_{i, j}=\frac{\partial H_{\text {Potts }}}{\partial S_{i, j}}=-J_{i, j}+2 \lambda_{i}^{C}\left[S_{i}-S_{i}^{0}\right]+2 \lambda_{j}^{C}\left[S_{j}-S_{j}^{0}\right]$

under the hypothesis that cell volumes cannot vary too much, which is biologically plausible due to homeostasis conditions.

Of course, surface tensions cannot be measured on confocal images, but the mechanical equilibrium condition gives us a relation between surface tensions and angles at contact points:

$$
\frac{\gamma_{A B}}{\sin \theta_{A B}}=\frac{\gamma_{A C}}{\sin \theta_{A C}}=\frac{\gamma_{B C}}{\sin \theta_{B C}}
$$

Thus, measuring the angles at each contact point give numerical relations between the surface tensions, and therefore, between the model parameters.

In practice, we take confocal images on control and mutant embryos at different developmental stages, where cell membranes have been stained. A semi-automated segmentation of the 3D confocal stacks is performed, in order to extract the precise position of all membranes. Cell volumes and interface areas are estimated. Contact points where 3 or 4 cells are in contact are visually detected, then the angles between tangents passing through the contact point are measured on the stacks using ImageJ $[1,20]$. The calibration procedure through the angle measurements at contact points has been validated on cellular Potts model simulations.

As the number of parameters is greater than the number of constraints, surface, volume and angular measurements are not sufficient to completely determine the model parametrisation, but the number of free parameters is significantly reduced. Measurements of the membrane curvatures may also be used to constraint the model parameters, but for the moment the data quality somehow limits the precision of the curvature information we can extract.

\section{Conclusion}

In order to better understand the role of mechanical interactions between cells during the first developmental stage of the mammal embryo, we developed a 3-dimensional mechanical model of the early mammalian embryo based on a cellular Potts model. This model is able to reproduce the main geometrical features (shape, cell arrangement) of the early mammalian embryo at its first developmental stages up to the 16-cell stage, with very simple mechanical hypotheses on cell elasticity and cell-cell adhesion. The model is robust and the geometrical features are relatively insensitive to small variations of the model parameters. We designed a calibration procedure based on confocal imaging data of embryos to increase the predictive power of the model. Once calibrated, our aim is to test its predictions against wild and mutant embryos at different development stages, and then to propose this model as a tool to help the study of biological hypotheses on the role of mechanics in mammal embryogenesis.

\section{References}

[1] Abramoff, M.D., Magelhaes, P.J. \& Ram, S.J. Image Processing with ImageJ, Biophotonics International, 11 (2004), no. 7, 36-42.

[2] Dard, N., Breuer, M., Maro, B., \& Louvet-Vallée, S. Morphogenesis of the mammalian blastocyst, Mol Cell Endocrinol, 282 (2008), 70-77.

[3] Dard, N., Le, T., Maro, B. \& Louvet-Vallée, S. Inactivation of aPKC $\lambda$ reveals a context dependent allocation of cell lineages in preimplantation mouse embryos, in preparation.

[4] Fleming, T.P. A quantitative analysis of cell allocation to trophectoderm and inner cell mass in the mouse blastocyst, Developmental Biology 119 (1987), 520-531.

[5] Glazier, J. A. \& Graner, F. Simulation of the differential adhesion driven rearrangement of biological cells, Phys. Rev. E 47 (1993), no. 3, 2128-2154.

[6] Graner, F. \& Glazier, J. A. Simulation of biological cell sorting using a two-dimensional extended potts model, Phys. Rev. Lett. 69 (1992), no. 13, 2013 2016.

[7] Graner, F. Can surface adhesion drive cellrearrangement? I: Biological cell-sorting, Journal of Theoretical Biology 164 (1993), no. 4, 455-476.

[8] Graner, F. Sawada Y. Can surface adhesion drive cell rearrangement? II: A geometrical model, Journal of Theoretical Biology 164 (1993), no. 4, 477506.

[9] Johnson, M.H., \& Ziomek, C.A. The foundation of two distinct cell lineages within the mouse morula, Cell 24 (1981), 71-80.

[10] Johnson, M.H., \& McConnell, J.M. Lineage allocation and cell polarity during mouse embryogenesis, Semin Cell Dev Biol 15 (2004), 583-597. 
[11] Kafer, J., Hayashi, T., Marée, A. F. M., Carthew, R. \& Graner, F. Cell adhesion and cortex contractility determine cell patterning in the Drosophila retina, Proceedings of the National Academy of Sciences 104 (2007), no. 47, 18549.

[12] Le Guillou, L. Bobbles, a Python module for Cellular Potts Models, http://lpnhe-simbiophys.in2p3. $\mathrm{fr} /,(2008-2009)$.

[13] Marée, A. F. M. From Pattern Formation to Morphogenesis: Multicellular Coordination in Dictyostelium discoideum, Ph.D. thesis, Utrecht University, 2000.

[14] Marée, A. F. M. \& Hogeweg, P. How amoeboids self-organize into a fruiting body: Multicellular coordination in Dictyostelium discoideum, Proceedings of the National Academy of Sciences 98 (2001), no. 7, 3879-3883.

[15] Maro, B., Gueth-Hallonet, C., Aghion, J., \& Antony, C. Cell polarity and microtubule organisation during mouse early embryogenesis, Development (1991), 17-25.

[16] Mintz, B. Experimental genetic mosaicism in the mouse. In Preimplantation stages of pregnancy,
Ciba Foundation Symposium, G.E.W. Wolstenholm, and M.O. Connor, eds. London: J+A Churchill (1965).

[17] Mombach, J. C. M. \& Glazier, J. A. Single cell motion in aggregates of embryonic cells, Phys. Rev. Lett. 76 (1996), no. 16, 3032-3035.

[18] Poplawski, N. J., Swat, M., Gens, J. S. \& Glazier, J. A. Adhesion between cells, diffusion of growth factors, and elasticity of the aer produce the paddle shape of the chick limb, Physica A: Statistical and Theoretical Physics 373 (2007), 521 - 532.

[19] Tarkowski, A.K. \& Wroblewska, J. Development of blastomeres of mouse eggs isolated at the 4- and 8cell stage. Journal of Embryology and experimental Morphology 18 (1967), 155-180.

[20] Rasband, W.S., ImageJ, U. S. National Institutes of Health, Bethesda, Maryland, USA, http://rsb.info. nih.gov/ij/, (1997-2008).

[21] Wolpert, L., Jessel, T., Lawrence, P., Meyerowitz, E., Robertson, E. \& Smith, J., Principles of Development, 3rd edn, Oxford University Press (2007). 